\title{
Some remarks on the coherent-state variational approach to nonlinear boson models
}

\author{
P Buonsante and V Penna \\ Dipartimento di Fisica and Unità C.N.I.S.M., Politecnico di Torino, C.so Duca degli \\ Abruzzi 24, I-10129 Torino, Italia
}

\begin{abstract}
The mean-field pictures based on the standard time-dependent variational approach have been widely used in the study of nonlinear many-boson systems such as the Bose-Hubbard model. The mean-field schemes relevant to Gutzwiller-like trial states $|F\rangle$, number-preserving states $|\xi\rangle$ and Glauber-like trial states $|Z\rangle$ are compared to evidence the specific properties of such schemes. After deriving the Hamiltonian picture relevant to $|Z\rangle$ from that based on $|F\rangle$, the latter is shown to exhibit a Poisson algebra equipped with a Weyl-Heisenberg subalgebra which preludes to the $|Z\rangle$-based picture. Then states $|Z\rangle$ are shown to be a superposition of $\mathcal{N}$-boson states $|\xi\rangle$ and the similarities/differences of the $|Z\rangle$-based and $|\xi\rangle$-based pictures are discussed. Finally, after proving that the simple, symmetric state $|\xi\rangle$ indeed corresponds to a $\mathrm{SU}(\mathrm{M})$ coherent state, a dual version of states $|Z\rangle$ and $|\xi\rangle$ in terms of momentum-mode operators is discussed together with some applications.
\end{abstract}

PACS numbers: 03.75.Fd, 03.65.Sq, 03.75Kk

\section{Introduction}

The semiclassical formulation of many-mode boson models based on the coherent-state (CS) method [1, 2] has proven to be an effective tool to describe the behavior of interacting bosons in many situations [3]-[11]. Such models, usually represented by a second-quantized Hamiltonian in terms of boson operators $a_{i}, a_{i}^{+}, n_{i}=a_{i}^{+} a_{i}$ with standard commutators $\left[a_{m}, a_{i}^{+}\right]=\delta_{m i}$, exhibit a dramatic complexity owing to their many-body nonlinear character. The combination of the CS method with the application of standard variational schemes allows one to circumvent this problem by reformulating model Hamiltonians into a mean-field $(\mathcal{M F})$ picture [6], [12] in which the Schrödinger problem for variational trial states $|\Phi\rangle=\left|\phi_{1}, \phi_{2} \ldots\right\rangle$ describing the system quantum state is reduced to a set of Hamilton equations governing the evolution of parameters $\phi_{j}$. A very standard choice [13] is $|\Phi\rangle \equiv|Z\rangle=\prod_{i}\left|z_{i}\right\rangle$ where, for each mode $a_{i}$, state $\left|z_{i}\right\rangle$ is a Glauber CS satisfying the defining equation $a_{m}\left|z_{m}\right\rangle=z_{m}\left|z_{m}\right\rangle$ and $\phi_{j}$ identify with CS parameters $z_{j}=\left\langle\Phi\left|a_{j}\right| \Phi\right\rangle$. Similar schemes have been developed for magnetic and multi-level atomic systems [6], [14] where $|\Phi\rangle$ is a product of spin CS. The important feature is that dynamical variables $\phi_{j}$ are, at the same time, the expectation 
values of the Hamiltonian operators and thus are providing information on microscopic physical processes. Significant examples are found within the physics of ultracold bosons confined in optical lattices where this $\mathcal{M F}$ formulation successfully describes complex dynamical behaviors [15]-[25]. There, CS parameters usually represent either the onsite superfluid order parameters of local condensates or the expectation values of local operators such as $a_{i}^{+} a_{m}$ and $n_{i}$ giving informations on space correlations and boson populations, respectively.

The most general version of this $\mathcal{M F}$ picture, however, is achieved by using a Gutzwiller-like [26] trial state $|\Phi\rangle$ exhibiting yet a factorized semiclassical form, but involving constituent states more structured than CS. These are

$$
|F\rangle=\prod_{i=1}^{M}\left|F_{i}\right\rangle=\prod_{i=1}^{M} \sum_{n_{i}=0}^{\infty} f_{n_{i}}^{i}|n\rangle_{i},
$$

where $\left|F_{i}\right\rangle$ has replaced local state $\left|z_{i}\right\rangle$ in $|Z\rangle, n_{i}|n\rangle_{i}=n|n\rangle_{i}$ and $M$ is the bosonmode number namely, for many models, the lattice-site number. This choice ensures an improved description of microscopic processes in the sense that, for each mode, infinitelymany variational parameters $f_{n_{i}}^{i}$ are available now in place of the $M$ parameters $z_{i}$ of $|Z\rangle$. The $|F\rangle$-based approach has been applied in studying the dynamics of Bose-Hubbard (BH) model [27], 28] as well as its zero-temperature critical properties [29]-32].

Recently, a third variational scheme has been considered [23], 25] to approach the dynamics of many-mode boson models, where state $|\Phi\rangle$ is assumed to have the form

$$
|\mathcal{N}, \xi\rangle=(\mathcal{N} !)^{-1 / 2}\left(A^{+}\right)^{\mathcal{N}}|0,0, \ldots, 0\rangle,
$$

where $A^{+}=\sum_{i=1}^{M} \xi_{i} a_{i}^{+}$and the constraint $\sum_{i=1}^{M}\left|\xi_{i}\right|^{2}=1$ ensuring its normalization. Different from states $|Z\rangle$ and $|F\rangle$, the distinctive property [33] of states $|\xi\rangle(\mathcal{N}$ will be often implied in $|\mathcal{N}, \xi\rangle)$ is to diagonalize, by construction, the boson-number operator $N=\sum_{i} n_{i}$ whose eigenvalue can be easily shown to identify with index $\mathcal{N}$. Hence, states $|\xi\rangle$ having $\mathcal{N}$ as a good quantum number naturally embody the property $[N, H]=0$ characterizing usually many-mode boson Hamiltonians $H$. This valuable feature reflects in turn an even important fact that states 2 actually coincide with the CS of group $\mathrm{SU}(\mathrm{M})$ where eigenvalue $\mathcal{N}$ is the index labeling the representation of $\mathrm{SU}(\mathrm{M})$.

The structure of formula 2, however, appears quite different from the (standard) group-theoretic form of $\mathrm{SU}(\mathrm{M})$ coherent states. The standard definition [1], in fact, states that $|\xi\rangle=g|\Omega\rangle$ with $g \in \mathrm{SU}(\mathrm{M})$ where $|\Omega\rangle$ is an appropriate extremal state. Then a CS is generated through the exponential action of an algebra element $a \in \operatorname{su}(\mathrm{M})$ such that $g=\exp (i a)$ where $a$ is in general a linear combination of $\mathrm{su}(\mathrm{M})$ generators. A well-known $M$ boson-mode realization [2] of SU(M) CS is, for example,

$$
g|\Omega\rangle=T(\zeta)|\Omega\rangle, \quad|\Omega\rangle=|0, \ldots, \mathcal{N}, 0, \ldots\rangle
$$

where $T(\zeta)=e^{i a}$ is the displacement operator, $a=\sum_{\ell \neq m}^{M}\left(\zeta_{\ell}^{*} a_{m}^{+} a_{\ell}+\zeta_{\ell} a_{\ell}^{+} a_{m}\right), \zeta_{\ell} \in \mathbf{C}$, and $n_{\ell}|\Omega\rangle=\delta_{\ell m} \mathcal{N}|\Omega\rangle$. Such a definition has been (and is) currently in use to represent quantum dynamical processes in microscopic systems within Quantum Optics, Condensed-Matter theory, and Nuclear Physics (see [2], 34] and references therein). 
Except for the case $M=2$, where states 2 are easily related [2] to definition $g|\Omega\rangle$ (see Appendix A), for $M \geq 3$ the connection of formula 2 with the group-theoretic form of $\mathrm{SU}(\mathrm{M}) \mathrm{CS}$ is less direct owing to the difficulty in disentangling the group element $g$ through the Baker-Campbell-Hausdorff decomposition [2]. As noted in [35], where this issue has been investigated, even if $\mathrm{SU}(2) \mathrm{CS}$ have been widely used in the literature, not much work has been devoted to find realizations of SU(M) CS more practicable in physical applications. In this respect, indeed definition 2 has proven to supply a useful tool in applications. However, despite states 2 being tacitly presented as CS of group $\mathrm{SU}(\mathrm{M})$ in various papers, their connection with the group-theoretic definition within CS Theory is far from being evident. We thus devote some attention to this particular aspect even if it might be known to authors [35]-[38], [39] involved on mathematical aspects of CS.

This paper is aimed at comparing the $\mathcal{M F}$ schemes based on states $|F\rangle,|Z\rangle$ and $|\xi\rangle$, widely used in applications to boson systems. We enlight some formal aspects concerning both the implementation of the (time-dependent) variational approach within such schemes, and the representation of trial states in terms of CS. We emphasize that some parts of our discussion have a review character and involve well-known theoretic tools. Nevertheless, a direct comparison among these three $\mathcal{M} \mathcal{F}$ schemes has never been presented to our knowledge. We feel that such a comparison can elucidate their specific properties and advantages at the operational level prompting as well their applications.

A first objective of this paper is to evidence how the variational schemes based on state $|F\rangle$ and $|Z\rangle$, respectively, are related to each other. In section 2, after reviewing the variational procedure that amounts to reformulating quantum Hamiltonian models in terms of effective Hamiltonians and of the corresponding dynamical equations, we focus on the formal derivation of the Hamiltonian picture relevant to $|Z\rangle$ from the one based on $|F\rangle$. We show that the Poisson algebra of variables $f_{n}^{j}, \bar{f}_{m}^{\ell}$ is naturally equipped with a (classical) Weyl-Heisenberg sub-algebra preluding to the $|Z\rangle$-based picture.

A second objective is to relate $\mathrm{SU}(\mathrm{M}) \mathrm{CS}|\xi\rangle$ to states $|Z\rangle$ and $|F\rangle$. In section 3 , following [13], we show that Glauber-like trial state

$$
|Z\rangle=\prod_{i=1}^{M}\left|z_{i}\right\rangle, \quad\left|z_{i}\right\rangle=e^{z_{i} a_{i}^{+}-z_{i}^{*} a_{i}}|0\rangle_{i}=e^{-\left|z_{i}\right|^{2} / 2} \sum_{n=0}^{\infty} \frac{z_{i}^{n}}{\sqrt{n !}}|n\rangle_{i}, \quad z_{i} \in \mathbf{C}
$$

can be expressed as a superposition of $\mathrm{SU}(\mathrm{M})$ states $|\xi\rangle$ thus making evident why the dynamical equations obtained in the $|Z\rangle$-based scheme have essentially the same form of those obtained in the $|\xi\rangle$-based scheme. To illustrate this situation we derive the $\mathcal{M F}$ dynamics relevant to the BH model showing (see Appendix D) how the use of form 2 in place of the standard $\mathrm{SU}(\mathrm{M}) \mathrm{CS}$ definition is extremely advantageous. In this section we also display an explicit way to relate state 2 with the standard form $|\xi\rangle=g|\Omega\rangle$ of CS theory involving one among $M$ possible (equivalent) choices of extremal vector $|\Omega\rangle$ and of the relevant maximal isotropy algebra. Finally, in section 4, we discuss the property of "duality" inherent in space-like states $|Z\rangle$ and $|\xi\rangle$ defined in terms of ambient-lattice operators $a_{i}^{+}$showing how they can be easily rewritten as momentum-like 
states involving momentum modes $b_{k}^{+}$. We exploit this property to construct Schrödinger cat states in terms of states $|\xi\rangle$ and to show that they exhibit specific momentum features.

\section{Mean-field approaches based on states $|F\rangle$ and $|Z\rangle$}

In order to compare the $|Z\rangle$-based approach with the $|F\rangle$-based approach we refer to the $\mathcal{M F}$ dynamical equations stemmed from such schemes for the well-known BoseHubbard Hamiltonian [6]-[10]. Optical-lattice confinement shows that real boson lattice systems are effectively described within the Bose-Hubbard picture. The corresponding Hamiltonian [40], defined on an $M$-site lattice, is

$$
H=\frac{U}{2} \sum_{j}\left(n_{j}^{2}-n_{j}\right)-\sum_{<j \ell} T_{j \ell} a_{j}^{+} a_{\ell}
$$

where $n_{i}=a_{i}^{+} a_{i}(i=1, \ldots, M)$ and $a_{i}, a_{i}^{+}$obey the standard commutators $\left[a_{m}, a_{i}^{+}\right]=\delta_{m i}$. In the hopping term $\sum_{<j \ell>} \equiv \frac{1}{2} \sum_{j} \sum_{\ell \in j}$, where $\ell$ labels the nearest neighbour sites of $j$, and $T_{j \ell}=T_{\ell j}$. This model well represents the boson tunneling among the potential wells forming $d$-dimensional arrays $(d=1,2,3)$ through the hopping amplitude $T_{j \ell}$, and takes into account boson-boson interactions by means of parameter $U$. For one-dimensional homogeneous arrays the hopping term reduces to $T \Sigma_{j}\left(a_{j+1}^{+} a_{j}+a_{j+1} a_{j}^{+}\right)$.

The $\mathcal{M F}$ dynamics relevant to trial state 1 is easily derived. State $|F\rangle=\Pi_{i}\left|F_{i}\right\rangle$, where $\left|F_{i}\right\rangle=\sum_{n} f_{n}^{i}|n\rangle_{i}$ and $|n\rangle_{i}$ is such that $a_{i}^{+}|n\rangle_{i}=\sqrt{1+n}|n+1\rangle_{i}, a_{i}|n\rangle_{i}=\sqrt{n}|n-1\rangle_{i}$, obeys the normalization condition $\langle F \mid F\rangle=\Pi_{i}\left[\sum_{n}\left|f_{n}^{i}\right|^{2}\right]=1$. The application of the time-dependent variational principle [2], [6] amounts to deriving dynamical equation for parameters $f_{n}^{i}$ by stationarizing the weak form of Schrödinger equation $\langle\Psi|S| \Psi\rangle=0$ where $S:=i \hbar \partial_{t}-H$. In order to illustrate this procedure, we write explicitly the expectation value of microscopic physical operators appearing in $H$. These are

$$
\alpha_{i}=\left\langle F\left|a_{i}\right| F\right\rangle=\sum_{m} \sqrt{m}\left\langle F_{i}\left|f_{m}^{i}\right| m-1\right\rangle=\sum_{m} \sqrt{m+1} \bar{f}_{m}^{i} f_{m+1}^{i},
$$

$\left\langle F\left|a_{i} a_{j}^{+}\right| F\right\rangle=\left\langle F_{i}\left|a_{i}\right| F_{i}\right\rangle\left\langle F_{j}\left|a_{j}^{+}\right| F_{j}\right\rangle=\alpha_{i} \alpha_{j}^{*}$, and $\left\langle F\left|\left(n_{i}\right)^{s}\right| F\right\rangle=\sum_{n} n^{s}\left|f_{n}^{i}\right|^{2}$ where the exponent $s$ is an integer. To calculate $\left\langle\Psi\left|\partial_{t}\right| \Psi\right\rangle$ and $\mathcal{H}=\langle\Psi|H| \Psi\rangle$ in $\langle\Psi|S| \Psi\rangle$ we standardly set $|\Psi\rangle=e^{i A}|F\rangle$, the phase $A$ representing the effective action within the variational procedure. The first quantity becomes $\left\langle\Psi\left|\partial_{t}\right| \Psi\right\rangle=i \dot{A}+\left\langle F\left|\partial_{t}\right| F\right\rangle$ with

$$
\left\langle F\left|\partial_{t}\right| F\right\rangle=\sum_{j} \sum_{n} \bar{f}_{n}^{j} \dot{f}_{n}^{j}=\frac{1}{2} \sum_{j} \sum_{n}\left[\bar{f}_{n}^{j} \frac{d f_{n}^{j}}{d t}-\frac{d \bar{f}_{n}^{j}}{d t} f_{n}^{j}\right]+\frac{d}{d t} \sum_{j} \sum_{n}\left|f_{n}^{j}\right|^{2}
$$

while $\mathcal{H}=\langle F|H| F\rangle$ reads

$$
\mathcal{H}=\frac{U}{2} \sum_{j}\left(\sum_{n}\left(n^{2}-n\right)\left|f_{n}^{j}\right|^{2}\right)-\sum_{<\ell j>} T_{\ell j} \alpha_{\ell} \alpha_{j}^{*}
$$

From the action $A=\int L d t=\int d t\left[i \hbar\left\langle F\left|\partial_{t}\right| F\right\rangle-\mathcal{H}\right]$ (in which the second term of

$\left\langle F\left|\partial_{t}\right| F\right\rangle$, a total time-derivative, can be eliminated) one obtains the Lagrange equations

$$
\frac{\partial}{\partial t} \frac{d L}{d \dot{\overline{f_{m}^{i}}}}-\frac{d L}{d \bar{f}_{m}^{i}}=0, \quad \frac{\partial}{\partial t} \frac{d L}{d \dot{f}_{m}^{i}}-\frac{d L}{d f_{m}^{i}}=0
$$


$\left(d f_{m}^{i} / d t=\dot{f_{m}^{i}}\right)$ that can equivalently be written as (we set $\left.\hbar=1\right)$

$$
-i \dot{f}_{m}^{i}+\frac{\partial \mathcal{H}}{\partial \bar{f}_{m}^{i}}=0, \quad i \dot{\overline{f_{m}^{i}}}+\frac{\partial \mathcal{H}}{\partial f_{m}^{i}}=0 .
$$

By defining the Poisson brackets

$$
\{A, B\}=-i \sum_{n} \sum_{j}\left[\frac{\partial A}{\partial f_{n}^{j}} \frac{\partial B}{\partial \bar{f}_{n}^{j}}-\frac{\partial B}{\partial f_{n}^{j}} \frac{\partial A}{\partial \bar{f}_{n}^{j}}\right]
$$

equations (6) can be formulated within the standard Hamiltonian formalism as $d f_{m}^{i} / d t=$ $\left\{f_{m}^{i}, \mathcal{H}\right\}$ and $d \bar{f}_{m}^{i} / d t=\left\{\bar{f}_{m}^{i}, \mathcal{H}\right\}$. The resulting $\mathcal{M F}$ dynamical equations are

$$
i \dot{f}_{m}^{i}=\frac{U}{2}\left(m^{2}-m\right) f_{m}^{i}-\sqrt{m+1} f_{m+1}^{i} \Phi_{i}^{*}-\sqrt{m} f_{m-1}^{i} \Phi_{i},
$$

(equations for $\bar{f}_{m}^{i}$ are obtained from the latter by complex conjugation), where

$$
\Phi_{i}^{*}=\sum_{j \in i} T_{i j} \alpha_{j}^{*}, \quad \Phi_{i}=\sum_{\ell \in i} T_{\ell i} \alpha_{\ell},
$$

and, in addition to definition 4, one has $\alpha_{i}^{*}=\sum_{m=0}^{\infty} \sqrt{m} \bar{f}_{m}^{i} f_{m-1}^{i}$. Concluding, we recall that within the $|F\rangle$-based scheme the average total particle number $\mathcal{N}=\langle F|N| F\rangle=$ $\sum_{i}\left\langle F\left|n_{i}\right| F\right\rangle=\sum_{i} \sum_{n} n\left|f_{i}^{n}\right|^{2}$ should be a constant of motion of Hamiltonian 5. The validity of this property is verified in Appendix C. In addition to $\mathcal{N}$, other $M$ motion constants can be shown to be represented by $I_{i}=\sum_{n}\left|f_{i}^{n}\right|^{2}=\left\langle F_{i} \mid F_{i}\right\rangle$. These allows one to implement the local-state normalization condition $\left\langle F_{i} \mid F_{i}\right\rangle=1$.

\subsection{Connection with the Glauber-like trial state scenario}

A simple assumption allows one to recover state $|Z\rangle$ from $|F\rangle$ and relate the corresponding variational schemes. This is

$$
f_{m}^{i}=e^{-\left|z_{i}\right|^{2} / 2} z_{i}^{m} / \sqrt{m !}
$$

entailing $\sum_{n=0}^{\infty} f_{n}^{i}|n\rangle_{i}=e^{-\left|z_{i}\right|^{2} / 2} \sum_{n=0}^{\infty}\left(a_{i}^{+}\right)^{n}|0\rangle_{i} / n$ ! and therefore

$$
|F\rangle=\prod_{i=1}^{M} \sum_{n=0}^{\infty} f_{n}^{i}|n\rangle_{i}=\prod_{i=1}^{M} e^{z_{i} a_{i}^{+}-z_{i}^{*} a_{i}}|0\rangle_{i}=\prod_{i=1}^{M}\left|z_{i}\right\rangle=|Z\rangle,
$$

where the defining formula of Glauber CS $|z\rangle=e^{z a^{+}-z^{*} a}|0\rangle=e^{-|z|^{2} / 2} e^{z a^{+}}|0\rangle$ has been used for each space mode together with the Baker-Campbell-Hausdorf decomposition formula [1]. The same assumption enables one to find the new form of parameter 4

$$
\alpha_{j}=\sum_{m=0}^{\infty} \sqrt{m+1} \bar{f}_{m}^{j} f_{m+1}^{j}=e^{-\left|z_{j}\right|^{2}} \sum_{m=0}^{\infty} z_{j} \frac{\left|z_{j}\right|^{2 m}}{m !}=z_{j},
$$

showing that $\alpha_{j}$ reduce to Glauber CS parameters $z_{j}$, and formulas $\left\langle F\left|a_{i} a_{j}^{+}\right| F\right\rangle=z_{i} z_{j}^{*}$, $\left\langle F\left|n_{i}\right| F\right\rangle=\left|z_{i}\right|^{2}$ and $\left\langle F\left|n_{i}^{2}\right| F\right\rangle=\left|z_{i}\right|^{4}+\left|z_{i}\right|^{2}$. In order to recover the $\mathcal{M F}$ equations inherent in the $|Z\rangle$-based picture we consider the time derivative of $\alpha_{j}$. This is given 
by $i \dot{\alpha}_{j}=\sum_{m=0}^{\infty} \sqrt{m+1}\left[i f_{m+1}^{j} d \bar{f}_{m}^{j} / d t+i \bar{f}_{m}^{j} d f_{m+1}^{i} / d t\right]$, which reduces to (the detailed calculation is carried out in Appendix B

$$
i \frac{d \alpha_{j}}{d t}=\sum_{m=0}^{\infty}\left[U m \sqrt{m+1} \bar{f}_{m}^{j} f_{m+1}^{j}\right]-\Phi_{j} .
$$

Notice that, as illustrated in Appendix B, no explicit assumption on the form of $f_{m}^{j}$ has been requested so far (except for $\left\langle F_{j} \mid F_{j}\right\rangle=1$ ) in getting 12. At this point, however, the use of formula 9 in 12 becomes necessary. We find $\sum_{m=0}^{\infty} U m \sqrt{m+1} \bar{f}_{m}^{j} f_{m+1}^{j}=$ $U z_{j} \sum_{m=0}^{\infty} m \bar{f}_{m}^{j} f_{m}^{j}=U z_{j}\left|z_{j}\right|^{2}$ which leads, in turn, to the well-known final equations

$$
i \dot{z}_{j}=U z_{j}\left|z_{j}\right|^{2}-\sum_{\ell \in j} T_{\ell j} z_{\ell}
$$

describing a set of discrete nonlinear Schröndinger equations [41], namely the $\mathcal{M F}$ dynamical equations associated to the Bose-Hubbard model within the Glauber-like variational picture [6]. Equations 13 can be issued from the new Hamiltonian

$$
\mathcal{H}=\frac{U}{2} \sum_{i}\left|z_{i}\right|^{4}-\sum_{<j \ell>} T_{j \ell} z_{j} z_{\ell}^{*}
$$

obtained by rewriting formula 5 in terms of $z_{j}$, and defining the new Poisson brackets (PB)

$$
\{A, B\}=-i \sum_{\ell}\left[\frac{\partial A}{\partial z_{\ell}} \frac{\partial B}{\partial \bar{z}_{\ell}}-\frac{\partial B}{\partial z_{\ell}} \frac{\partial A}{\partial \bar{z}_{\ell}}\right] \Leftrightarrow\left\{z_{j}, z_{\ell}^{*}\right\}=-i \delta_{j \ell} .
$$

The crucial point that explains and justifies the whole reduction of the Hamiltonian picture based variables $f_{n}^{i}, \bar{f}_{m}^{\ell}$ to the one involving the restricted set of variables $z_{i}, \bar{z}_{\ell}$ thus consists in showing that PB 15 are consistent with PB of 7. In particular, $\alpha_{i}, \alpha_{\ell}^{*}$ must be shown to exhibit, within algebra 7 , the same algebraic structure of variables $z_{j}, z_{\ell}^{*}$. By setting $A=\alpha_{i}$ and $B=\alpha_{\ell}^{*}$ in PB 7 one discovers that

$$
\begin{aligned}
& \quad\left\{\alpha_{j}, \alpha_{\ell}^{*}\right\}=\sum_{m} \sum_{n} \sqrt{m+1} \sqrt{n+1}\left\{\bar{f}_{m}^{j} f_{m+1}^{j}, f_{n}^{\ell} \bar{f}_{n+1}^{\ell}\right\}= \\
& =-i \delta_{j \ell} \sum_{m}(m+1)\left(\left|f_{m}^{j}\right|^{2}-\left|f_{m+1}^{j}\right|^{2}\right)=-i \delta_{j \ell} \sum_{m}\left|f_{m}^{j}\right|^{2}=-i \delta_{j \ell}
\end{aligned}
$$

due to normalization $\left\langle F_{i} \mid F_{i}\right\rangle=1$. Also, one easily proves that $\left\{\alpha_{j}, N_{\ell}\right\}=-i \delta_{j \ell} \alpha_{j}$, where $N_{\ell}=\left\langle F\left|n_{\ell}\right| F\right\rangle=\sum_{n} n\left|f_{n}^{\ell}\right|^{2}$. Hence, it is an intrinsic feature of algebra 7 characterized by $\left\{f_{n}^{j}, \bar{f}_{m}^{\ell}\right\}=-i \delta_{j \ell} \delta_{n m}$ the property that $\alpha_{i}, \alpha_{\ell}^{*}$ form a (classical) Weyl-Heisenberg sub-algebra of algebra 7. Noticeably, the latter represents the classical counterpart of the original boson algebra $\left[a_{j}, n_{\ell}\right]=\delta_{j \ell} a_{j},\left[a_{j}, a_{\ell}^{+}\right]=\delta_{j \ell}$ charaterizing Hamiltonian 3 , Then identities $\alpha_{j} \equiv z_{j}$, and $\alpha_{\ell}^{*} \equiv z_{\ell}^{*}$, obtained by assuming $f_{m}^{j}$ as a function of $z_{j}$ (see formula 11), quite naturally entail that $z_{j}, z_{\ell}^{*}$ obey the canonical brackets given in 15 within the Glauber-like scheme. This completes the proof that the $|Z\rangle$-based variational picture is consistently contained within that based on the more structured state $|F\rangle$.

Concluding, we notice that if an effective Hamiltonian $\mathcal{H}$ depending on $f_{n}^{i}, \bar{f}_{m}^{\ell}$ can be rewritten in terms of collective variables $\alpha_{j}, \alpha_{\ell}^{*}$ then sub-algebra [16 is sufficient for determining the evolution of the system and the $|F\rangle$-based picture becomes redundant. 
This is not the case of Hamiltonian 5 where, owing to the presence of the nonlinear $U$-dependent term, algebra 7 is necessary to derive the relevant motion equations.

Comparing equations 8 and 13 fully evidences how the more pronounced quantum character of the $|F\rangle$-based picture involves a dynamical scenario of great complexity. The marked semiclassical character of the $|Z\rangle$-based picture instead appears when comparing quantum model 3 with Hamiltonian 14. The latter, in fact, is essentially obtained from 3 through the substitutions $a_{i} \rightarrow z_{i}, a_{i}^{+} \rightarrow \bar{z}_{i}$, namely by implementing the Bogoliubov approximation. At a formal level, the $|Z\rangle$-based scheme thus provides an effective, dynamically-consistent formulation of the Bogoliubov semiclassical picture.

\section{Mean-field approach based on state $|\xi\rangle$}

A quite significant form of state $|Z\rangle$ given by 10 is achieved with a simple calculation

$$
\begin{aligned}
& |Z\rangle=\prod_{i=1}^{M}\left|z_{i}\right\rangle=e^{-\frac{1}{2} \sum_{i}\left|z_{i}\right|^{2}} \prod_{i=1}^{M} e^{z_{i} a_{i}^{+}}|0\rangle_{i}=e^{-\sum_{i}\left|z_{i}\right|^{2} / 2} e^{\sum_{i} z_{i} a_{i}^{+}}|0\rangle_{i}= \\
& =e^{-\frac{1}{2} \sum_{i}\left|z_{i}\right|^{2}} \sum_{S=0}^{\infty} \frac{1}{S !}\left(\sum_{i} z_{i} a_{i}^{+}\right)^{S}|0,0 \ldots 0\rangle=e^{-\mathcal{N} / 2} \sum_{S=0}^{\infty} \frac{\mathcal{N}^{\frac{S}{2}}}{\sqrt{S !}}|S ; \xi\rangle,
\end{aligned}
$$

where $\Pi_{i=1}^{M}|0\rangle_{i}=|0,0, \ldots 0\rangle, \xi_{i}=z_{i} / \sqrt{\mathcal{N}}$, and $|S ; \xi\rangle$ corresponds to state $|\xi\rangle$ defined by 2 where the group-representation index $S$ has been evidenced. State $|S ; \xi\rangle$ is characterized by $\langle\xi ; S|N| S ; \xi\rangle=S$ and the orthogonality property $\left\langle\zeta ; S^{\prime} \mid S ; \xi\right\rangle=\delta_{S S^{\prime}}$. The final version of $|Z\rangle$ derives from the observation that $\langle Z|N| Z\rangle=\sum_{i}\left|z_{i}\right|^{2}=\mathcal{N}$ is the average total boson number in $|Z\rangle$-based scheme and that $\xi_{i}=z_{i} / \sqrt{\mathcal{N}}$ is consistent with the normalization condition $\sum_{i}\left|\xi_{i}\right|^{2}=1$ of SU(M) coherent states. The latter follows from the scalar-product formula of two CS states $|\xi\rangle$ and $|\eta\rangle$ given by $\langle\eta \mid \xi\rangle=\left(\Sigma_{i} \eta_{i}^{*} \xi_{i}\right)^{S}$.

The new information about $|Z\rangle$ is therefore that states $|S ; \xi\rangle$ are its constitutive elements. In particular, state 2 features the property of incorporating only contributions of Fock states pertaining to the $S$-particle sectors of the Hilbert space. This becomes quite evident rewriting $|S ; \xi\rangle$ as

$$
|S ; \xi\rangle=\frac{1}{\sqrt{S !}}\left(\sum_{i} \xi_{i} a_{i}^{+}\right)^{S}|0\rangle=\sum_{\vec{m}}^{(S)} \frac{\sqrt{S !}}{\sqrt{\prod_{i}\left(m_{i} !\right)}} \xi_{1}^{m_{1}} \ldots \xi_{M}^{m_{M}}|\vec{m}\rangle
$$

where $|0\rangle=|0,0 \ldots 0\rangle$ and superscript $(S)$ recalls that $S=\sum_{i} m_{i}$ and $|\vec{m}\rangle$ is such that

$$
|\vec{m}\rangle=\left|m_{1}, \ldots m_{M}\right\rangle=\prod_{i}^{(S)} \frac{\left(a_{i}^{+}\right)^{m_{i}}}{\sqrt{m_{i} !}}|0,0 \ldots 0\rangle \quad \Rightarrow \quad N|\vec{m}\rangle=\sum_{i} m_{i}|\vec{m}\rangle=S|\vec{m}\rangle .
$$

The previous formulas allows one to evaluate the weight of state $|L ; \zeta\rangle$ in $|Z\rangle$

$$
\langle\zeta ; L \mid Z\rangle=e^{-\mathcal{N} / 2} \sum_{S=0}^{\infty} \frac{\mathcal{N}^{S / 2}}{\sqrt{S !}}\langle\zeta ; L \mid S ; \xi\rangle=e^{-\frac{\mathcal{N}}{2}} \frac{\mathcal{N}^{\frac{L}{2}}}{\sqrt{L !}}\left(\sum_{i} \zeta_{i}^{*} \xi_{i}\right)^{L} .
$$


Upon setting $\zeta=\xi$, the normalization condition $\sum_{i}\left|\xi_{i}\right|^{2}=1$ entails that $\langle\zeta ; L \mid Z\rangle=$ $e^{-\mathcal{N} / 2} \mathcal{N}^{L / 2} / \sqrt{L !}$ whose maximum value is reached for $L \equiv \mathcal{N}(\mathcal{N}$ is assumed to be integer). Considering $|L ; \xi\rangle$ with $L=\mathcal{N} \pm p$ and $p<<L$, one easily finds that the state-weight distribution around the maximum-weight state $|\mathcal{N} ; \xi\rangle$ is not sharp.

The variational procedure reviewed in section 2 can once more be applied to $\mathrm{BH}$ model 3 assuming $|\Psi\rangle=e^{i A}|\xi\rangle$ as the trial state. The weak form of Schrödinger equation $\left\langle\Psi\left|\left(i \hbar \partial_{t}-H\right)\right| \Psi\right\rangle=0$ provides the action $A=\int d t \mathcal{L}(\xi)$ where the effective Lagrangian $\mathcal{L}(\xi)=i \hbar\left\langle\xi\left|\partial_{t}\right| \xi\right\rangle-\langle\xi|H| \xi\rangle$ supplies the dynamical equations of variables $\xi_{i}$. The calculation of both $\left\langle\xi\left|\partial_{t}\right| \xi\right\rangle$ and $\mathcal{H}(\xi)=\langle\xi|H| \xi\rangle$ is carried out in Appendix D together with the basic formulas required to achieve these results. We find, in particular, that the average local boson number is $\left\langle\xi\left|n_{i}\right| \xi\right\rangle=\mathcal{N}\left|\xi_{j}\right|^{2}$, reproducing consistently $\langle\xi|N| \xi\rangle=\mathcal{N} \sum_{i}\left|\xi_{j}\right|^{2}=\mathcal{N}$. This suggests to define variables $\psi_{j}=\sqrt{N} \xi_{j}$ (formally coinciding with $z_{j}$ ) for better comparing the present $\mathcal{M F}$ dynamics with that issued from trial state $|Z\rangle$. Explicitly, one finds $\left\langle\xi\left|\partial_{t}\right| \xi\right\rangle=\mathcal{N} \sum_{j} \dot{\xi}_{j} \xi_{j}^{*}=\sum_{j} \dot{\psi}_{j} \psi_{j}^{*}$ and

$$
\mathcal{H}(\xi)=\langle\xi|H| \xi\rangle=\frac{U(\mathcal{N}-1)}{2 \mathcal{N}} \sum_{j}\left|\psi_{j}\right|^{4}-\sum_{<j \ell>} T_{j \ell} \psi_{j}^{*} \psi_{\ell}
$$

while the dynamics is found to be governed by

$$
i \frac{d \psi_{j}}{d t}=U \frac{(\mathcal{N}-1)}{\mathcal{N}}\left|\psi_{j}\right|^{2} \psi_{j}-\sum_{j \in \ell} T_{j \ell} \psi_{\ell} .
$$

Equations 18 can be interpreted as the projection of equations 13 on a given $S$-particle Hilbert-space sector. In order to prove this property one has to consider the variational scheme based on a generic state $|\psi\rangle=\sum_{S} C_{S}|S ; \xi\rangle$. The latter reproduces state $|Z\rangle$ when the condition $C_{S}=(S !)^{-1 / 2} e^{-\mathcal{N} / 2} \mathcal{N}^{S / 2}$ is imposed. The $|\psi\rangle$-based scheme would involve, in this case, the effective Lagrangian $\mathcal{L}=i \hbar\left\langle\psi\left|\partial_{t}\right| \psi\right\rangle-\langle\psi|H| \psi\rangle$ which, in the case $|\psi\rangle=|Z\rangle$, leads to equations 13. Observing that $\langle\xi ; R \mid S ; \xi\rangle=\delta_{R S}$, then

$$
\langle\psi|X| \psi\rangle=\sum_{R} \sum_{S} C_{R} C_{S}\langle\xi ; R|X| S ; \xi\rangle=\sum_{S} C_{S}^{2}\langle\xi ; S|X| S ; \xi\rangle,
$$

for both $X=H$ and $X=\partial_{t}$, states $\partial_{t}|S ; \xi\rangle$ and $H|S ; \xi\rangle$ pertaining to the $S$-particle sector. Hence, $\mathcal{L}$ reduces to a summation $\mathcal{L}=\sum_{S} C_{S}^{2} \mathcal{L}_{S}(\xi)$ over independent $S$-particle Lagrangians $\mathcal{L}_{S}(\xi)=\left\langle\xi ; S\left|\left[i \hbar \partial_{t}-H\right]\right| S ; \xi\right\rangle$, the case $S=\mathcal{N}$ giving equations 18 .

Formally, no significant difference therefore distinguishes equations 18 (and the relevant generating Hamiltonian) from the picture corresponding to equations 13 if $(\mathcal{N}-1) / \mathcal{N} \rightarrow 1$ namely for boson numbers $\mathcal{N}$ sufficiently large. A profound difference instead concerns the role of variables $z_{i}$ and $\psi_{i}$ in the relevant schemes. While $z_{i}=\left\langle Z\left|a_{i}\right| Z\right\rangle$ relates $a_{i}$ to the local superfluid parameter $z_{i}$, its counterpart in the $|\xi\rangle$ based scheme has no explicit physical meaning since $\left\langle\xi\left|a_{i}\right| \xi\right\rangle=0$. State $a_{i}|\xi\rangle$ belongs in fact to the $(\mathcal{N}-1)$-particle Hilbert-space sector thus resulting othogonal to the $\mathcal{N}$ particle state $|\xi\rangle$. With state $|Z\rangle$ this effect is avoided since $|Z\rangle$ is spread on the whole Hilbert space. The equivalence between the two schemes is restored in the case of twoparticle operators $z_{i} z_{j}^{*}=\left\langle Z\left|a_{i} a_{j}^{+}\right| Z\right\rangle$ being comparable with $\psi_{i} \psi_{j}^{*}=\mathcal{N} \xi_{i} \xi_{j}^{*}=\left\langle\xi\left|a_{i} a_{j}^{+}\right| \xi\right\rangle$. 
Of course case $i=j$ describing local populations $\left\langle\Phi\left|n_{i}\right| \Phi\right\rangle, \Phi=Z, \xi$ is also included. Variables $\psi_{j}=\left|\psi_{j}\right| e^{i \theta_{j}}$ thus acquire a physical meaning in terms of local populations $\left\langle\xi\left|n_{i}\right| \xi\right\rangle=\left|\psi_{i}\right|^{2}$. The relevant phases $\theta_{j}$ have no role unless one considers expectation values of operators $a_{i} a_{j}^{+}$involving phase differences $\theta_{i}-\theta_{j}$.

\subsection{Group-theoretic form of state $|\xi\rangle$}

State 2 displays the particularly nice property to possess a fully symmetric structure mirroring the fact that all modes $a_{m}$ play an equal important role in defining $|\xi\rangle$. This symmetry must be "broken" for proving that 2 has the standard group-theoretic CS form where $|\xi\rangle$ is generated by the group action on an extremal state (the choice of the latter entails the loss of the symmetric form). To show this our first step consists in proving that formula 2 can be rewritten as

$$
|\xi\rangle=(\mathcal{N} !)^{-1 / 2}\left(A^{+}\right)^{\mathcal{N}}|0,0, \ldots, 0\rangle=(\mathcal{N} !)^{-1 / 2} E\left(a_{1}^{+}\right)^{\mathcal{N}} E^{+}|0,0, \ldots, 0\rangle
$$

where $E^{+}=E^{-1}$ and $E$ is an element of $\mathrm{SU}(\mathrm{M})$ whose parametrization in terms of variables $\xi_{i}$ can be easily determined. The action of $a_{i}^{+}$on the zero-boson Fock state $|0\rangle:=|0,0, \ldots, 0\rangle$ is the standard one $\left(a_{i}^{+}\right)^{p}|0,0, \ldots, 0\rangle=\sqrt{n_{i} !}\left|\ldots, 0, n_{i}, \ldots\right\rangle$ with $n_{i}=p$ while $a_{i}|0\rangle=0$. We point out that the choice of generating $A^{+}$from $a_{m}^{+}$rather than from $a_{1}^{+}$is equally possible and simply entails choosing, in turn, one from $M$ possible parametrizations for $|\xi\rangle$ and the relevant form of $E$. This arbitrariness reflects the just mentioned symmetry of formula 2. For proving 19 we show that $E a_{1}^{+} E^{+}=A^{+}$where $E=e^{i S} e^{i D}$ is defined as

$$
S=\sum_{i=1}^{M} \phi_{i} n_{i}, \quad D=\sum_{i=2}^{M} \theta_{i}\left(a_{1}^{+} a_{i}+a_{i}^{+} a_{1}\right), \quad\left(D^{+}=D\right)
$$

with $\phi_{i} \in \mathbf{R}$ and $\theta_{i} \in \mathbf{R}$. Upon setting $\sum_{k=2}^{M} \theta_{k}^{2}:=\theta^{2}$, standard calculations show that $e^{i D} a_{1}^{+} e^{-i D}=\sum_{j=1}^{M} y_{j} a_{j}^{+}$(see Appendix E) with $y_{1}=\cos \theta$ and $y_{k}=i \theta_{k} \sin \theta / \theta$ if $k \neq 1$. A further action of $e^{i S}$ gives

$$
e^{i S} e^{i D} a_{1}^{+} e^{-i D} e^{-i S}=\sum_{j=1}^{M} y_{j} e^{i \phi_{j}} a_{j}^{+}=\sum_{j=1}^{M} \xi_{j} a_{j}^{+}=A^{+},
$$

where $\xi_{1}=e^{i \phi_{1}} \cos \theta, \xi_{k}=i \theta_{k} e^{i \phi_{k}} \sin \theta / \theta$ and the action of factor $e^{i \phi_{i} n_{i}}$ in $e^{i S}=\Pi_{i} e^{i \phi_{i} n_{i}}$ is described by $e^{i \phi_{\ell} n_{\ell}} a_{\ell}^{+} e^{-i \phi_{\ell} n_{\ell}}=e^{i \phi_{\ell}} a_{\ell}^{+}$. The identification $A^{+}=E a_{1}^{+} E^{+}$suggested by formula 20 is confirmed by the fact that the correct normalization of $A^{+}$components $\xi_{j}$ follows from $\sum_{j=1}^{M}\left|\xi_{j}\right|^{2}=\cos ^{2} \theta+\sum_{k=2}^{M}\left(\theta_{k}^{2} / \theta^{2}\right) \sin ^{2} \theta=1$. Since $a_{m}^{+} a_{i}|0\rangle=n_{i}|0\rangle=0$ and thus $S|0\rangle=D|0\rangle=0$, formula 19 becomes

$$
|\xi\rangle=(\mathcal{N} !)^{-1 / 2} E\left(a_{1}^{+}\right)^{\mathcal{N}} E^{+}|0\rangle=(\mathcal{N} !)^{-1 / 2} E\left(a_{1}^{+}\right)^{\mathcal{N}}|0\rangle=E|\mathcal{N}, 0, \ldots, 0\rangle,
$$

being $E^{+}|0\rangle=e^{-i D} e^{-i S}|0\rangle=|0\rangle$ and $\left(a_{1}^{+}\right)^{\mathcal{N}}|0\rangle=\sqrt{\mathcal{N} !}|\mathcal{N}, 0, \ldots\rangle$. Upon observing that $E=e^{i S} e^{i D}=\exp \left[e^{i S} i D e^{-i S}\right] e^{i S}$ state $|\xi\rangle$ can be rewritten as

$$
|\xi\rangle=e^{i \phi_{1} \mathcal{N}} \exp \left[e^{i S} i D e^{-i S}\right]|\mathcal{N}, 0, \ldots, 0\rangle=e^{i \phi_{1} \mathcal{N}} T(\zeta)|\mathcal{N}, 0, \ldots, 0\rangle,
$$


where $e^{i S} a_{\ell}^{+} a_{1} e^{-i S}=e^{i\left(\phi_{\ell}-\phi_{1}\right)} a_{\ell}^{+} a_{1}$ entails that

$$
e^{i S} D e^{-i S}=\sum_{\ell=2}^{M}\left(\zeta_{\ell}^{*} a_{1}^{+} a_{\ell}+\zeta_{\ell} a_{\ell}^{+} a_{1}\right), \quad T(\zeta):=e^{i \sum_{\ell=2}^{M}\left(\zeta_{\ell}^{*} a_{1}^{+} a_{\ell}+\zeta_{\ell} a_{\ell}^{+} a_{1}\right)},
$$

with $\zeta_{\ell}=\theta_{\ell} e^{i\left(\phi_{\ell}-\phi_{1}\right)}, \ell=2,3 \ldots, M$. Summarizing, we have found that

$$
|\xi\rangle=\frac{1}{\sqrt{\mathcal{N} !}}\left(A^{+}\right)^{\mathcal{N}}|0,0, \ldots, 0\rangle=e^{i \phi_{1} \mathcal{N}} T(\zeta)|\mathcal{N}, 0, \ldots, 0\rangle,
$$

where $T(\zeta)$ is an element of group $\mathrm{SU}(\mathrm{M})$, which proves that state 2, up to an irrelevant phase factor, is generated by the group action of $T(\zeta)$. The identification of $T(\zeta)$ with an element of $\mathrm{SU}(\mathrm{M})$ is discussed in Appendix F. By setting $\phi_{1}=0$, the relation between $\xi_{i}$ and $\zeta_{\ell}$ is described by $\xi_{\ell}=\zeta_{\ell} \sin \theta / \theta$ whereas $\xi_{1}$ is fixed by $\left|\xi_{1}\right|^{2}=1-\sum_{\ell=2}^{M}\left|\xi_{\ell}\right|^{2}$. An initial choice with $A^{+}=E a_{m}^{+} E^{+}$in formula 19 would have entailed $T(\zeta)$ generated by $\sum_{\ell \neq m}^{M}\left(\zeta_{\ell}^{*} a_{m}^{+} a_{\ell}+\zeta_{\ell} a_{\ell}^{+} a_{m}\right)$ and the extremal state $|0, \ldots \mathcal{N}, \ldots\rangle=(\mathcal{N} !)^{-1 / 2}\left(a_{m}^{+}\right)^{\mathcal{N}}|0\rangle$.

As a final step, we prove that formula 21 is consistent with the group-theoretic definition of CS based on the notion of maximal isotropy subalgebra (MIS). Within the CS Theory [1] a class of CS is derived by identifying the (complex) MIS $\mathcal{B}$ of $\mathcal{G}=$ $\mathrm{su}(\mathrm{M})$ and the related extremal vector $\left|\psi_{0}\right\rangle$. The defining formula for $\left|\psi_{0}\right\rangle$ states that $a\left|\psi_{0}\right\rangle=\lambda_{a}\left|\psi_{0}\right\rangle, \lambda_{a} \in \mathbf{C}, \forall a \in \mathcal{G}_{0}$ where $\mathcal{B} \cap \mathcal{G}:=\mathcal{G}_{0}$. The (complex) MIS naturally related to formula 21 is given by

$$
\mathcal{B}=\left\{h_{k}, a_{1}^{+} a_{k}, a_{\ell}^{+} a_{k}(k \neq \ell): k, \ell \in[2, M]\right\}, \quad([\mathcal{B}, \mathcal{B}] \subseteq \mathcal{B})
$$

whose generators are such that $a_{1}^{+} a_{k}|\mathcal{N}, 0, \ldots, 0\rangle=a_{\ell}^{+} a_{k}|\mathcal{N}, 0, \ldots, 0\rangle=0$ and generators $h_{k}$ form the Cartan (abelian) subalgebra. The vector satisfying the defining formula for $\left|\psi_{0}\right\rangle$ is thus $|\mathcal{N}, 0, \ldots, 0\rangle$. According to the MIS scheme, coherent states are generated by the action on $\left|\psi_{0}\right\rangle$ of the elements of the quotient group $G^{c} / B$ where $G^{c}=\exp \mathcal{G}$ and $B=\exp \mathcal{B}$. The algebra that generates $G^{c} / B$ is in our case $\left\{a_{k}^{+} a_{1}: k \in[2, M]\right\}$, which entails that a coherent state, up to a normalization factor $\lambda$, has the form

$$
\lambda e^{\sum_{k} \eta_{k} a_{k}^{+} a_{1}}|\mathcal{N}, 0, \ldots, 0\rangle, \quad e^{\sum_{k} \eta_{k} a_{k}^{+} a_{1}} \in G^{c} / B .
$$

State 21 precisely has this form. In order to check this property, one can observe that $T(\zeta)=\exp \left[i \sum_{k=2}^{M}\left(\zeta_{k}^{*} a_{1}^{+} a_{k}+\zeta_{k} a_{k}^{+} a_{1}\right)\right]=e^{i \theta\left(a_{1}^{+} D+D^{+} a_{1}\right)}$, with $D=\sum_{k=2}^{M} \zeta_{k}^{*} a_{k} / \theta$ where

$$
\left[D, D^{+}\right]=\sum_{k=2}^{M} \sum_{\ell=2}^{M} \frac{\zeta_{k}^{*} \zeta_{\ell}}{\theta^{2}}\left[a_{k}, a_{\ell}^{+}\right]=\sum_{k=2}^{M} \frac{\left|\zeta_{k}\right|^{2}}{\theta^{2}}=1 .
$$

The exponent of $T(\zeta)$ can thus be viewed as an element of $\operatorname{su}(2)$ in the two-boson (Schwinger) realization with generators $J_{-}=a_{1}^{+} D, J_{+}=a_{1} D^{+}, J_{3}=\left(D^{+} D-a_{1}^{+} a_{1}\right) / 2$ and commutators $\left[J_{3}, J_{ \pm}\right]= \pm J_{ \pm}$and $\left[J_{+}, J_{-}\right]=2 J_{3}$. This information allows us to apply the standard decomposition formula $e^{v J_{+}-v^{*} J_{-}}=e^{u J_{+}} e^{\ln \left(1+|u|^{2}\right)} e^{-u^{*} J_{-}}$for the $\mathrm{SU}(2)$ elements [1] where $v, u \in \mathbf{C}, v=|v| e^{i \psi}, u=|u| e^{i \psi}$ and $|u|=\operatorname{tg}|v|$. Setting $v=i \theta$, which entails $u=i \operatorname{tg} \theta$, one has $T(\zeta)=e^{i \theta\left(D_{1}^{+} B+D^{+} a_{1}\right)}=e^{i \theta\left(J_{-}+J_{+}\right)}$thus obtaining

$$
T(\zeta)|\mathcal{N}, 0, \ldots, 0\rangle=\frac{e^{u J_{+}}}{\left(1+|u|^{2}\right)^{\mathcal{N}}}|\mathcal{N}, 0, \ldots, 0\rangle=\frac{e^{\sum_{k=2}^{M} \eta_{k} a_{k}^{+} a_{1}}}{\left(1+|u|^{2}\right)^{\mathcal{N}}}|\mathcal{N}, 0, \ldots, 0\rangle
$$


where $\eta_{k}=u \zeta_{k} / \theta=i e^{i \theta_{k}} \operatorname{tg} \theta$, and $J_{3}|\mathcal{N}, 0, \ldots, 0\rangle=(\mathcal{N} / 2)|\mathcal{N}, 0, \ldots, 0\rangle$ has been used together with $J_{-}|\mathcal{N}, 0, \ldots, 0\rangle=a_{1}^{+} D|\mathcal{N}, 0, \ldots, 0\rangle=0$. Therefore state 21 indeed can be cast into the CS form 22 determined within the theory of CS.

\section{The duality property of states $|Z\rangle$ and $|\xi\rangle$}

Both state $|Z\rangle$ and $|\xi\rangle$, whose definition involves boson operators $a_{j}$ and $a_{j}^{+}$of the ambient lattice, can be shown exhibiting a dual character which becomes evident when space-like operators are expressed as momentum-like operators through Fourier formulas

$$
b_{q}=\sum_{j=1}^{M} \frac{e^{-i \tilde{q} j}}{\sqrt{M}} a_{j}, \quad a_{j}=\sum_{j=1}^{M} \frac{e^{i \tilde{q} j}}{\sqrt{M}} b_{q}, \quad \tilde{q}:=2 \pi q / M, q \in[1, M]
$$

where $\left[a_{j}, a_{\ell}^{+}\right]=\delta_{j \ell}$ implies that $\left[b_{q}, b_{p}^{+}\right]=\delta_{q p}$. Notice that we assume periodic boundary conditions (namely the lattice is a closed ring) so that displacements $q \rightarrow q+s M$ and $j \rightarrow j+r M$ ( $r, s$ are integer) leave operators $a_{j}$ and $b_{q}$ unchanged. This condition, standardly assumed to simplify theoretical models, becomes necessary in real lattices with a ring geometry [42]. Concerning state $|Z\rangle=\prod_{j}\left|z_{j}\right\rangle$ simple calculations yield

$$
|Z\rangle=e^{-\frac{1}{2} \sum_{j}\left|z_{j}\right|^{2}} e^{\sum_{j} z_{j} a_{j}^{+}}|0\rangle=e^{-\frac{1}{2} \sum_{k}\left|v_{k}\right|^{2}} e^{\sum_{k} v_{k} b_{k}^{+}}|0\rangle=\prod_{k}\left|v_{k}\right\rangle=|V\rangle
$$

where $a_{\ell}|0\rangle=0=b_{k}|0\rangle$ has been used (recall that $|0\rangle=|0,0, \ldots, 0\rangle$ ) and, thanks to definitions 23, one has $v_{k}=\sum_{j=1}^{M} e^{-i \tilde{q} j} z_{j} / \sqrt{M}$, and $z_{j}=\sum_{j=1}^{M} e^{i \tilde{q} j} v_{q} / \sqrt{M}$. Trial states $|Z\rangle$ are thus equivalent to states $|V\rangle$ formed by momentum-like Glauber CS $\left|v_{k}\right\rangle=e^{v_{k} b_{k}^{+}-v_{k}^{*} b_{k}}|0\rangle_{k}$. Similarly, states $|\xi\rangle$ transform into momentum-like SU(M) CS

$$
|\mathcal{N}, \xi\rangle=\frac{\left(A^{+}\right)^{\mathcal{N}}}{\sqrt{\mathcal{N} !}}|0\rangle=\frac{\left(B^{+}\right)^{\mathcal{N}}}{\sqrt{\mathcal{N} !}}|0\rangle=|\mathcal{N}, \alpha\rangle, \quad \xi_{j}=\sum_{k=1}^{M} \frac{e^{i \tilde{k} j}}{\sqrt{M}} \alpha_{k}
$$

where the latter definition ensures $B^{+}=\sum_{k=1}^{M} \alpha_{k} b_{k}^{+} \equiv \sum_{j=1}^{M} \xi_{j} a_{j}^{+}=A^{+}$. Also, the counterpart of formula [17] in the momentum picture is easily derived

$$
|\mathcal{N} ; \alpha\rangle=\sum_{\vec{p}}^{(\mathcal{N})} C_{\vec{p}}(\mathcal{N}) \alpha_{1}^{p_{1}} \ldots \alpha_{M}^{p_{M}}|\vec{p}\rangle,
$$

where $C_{\vec{p}}(\mathcal{N}):=\left[\mathcal{N} ! / \prod_{k} p_{k} !\right]^{1 / 2}$ while $|\vec{p}\rangle=\left[\prod_{k} p_{k} !\right]^{-1 / 2} \prod_{k}\left(b_{k}^{+}\right)^{p_{k}}|0\rangle$ are momentum Fock states. While space-like states $|Z\rangle$ and $|\xi\rangle$ provide information on the local boson population by means of $\left\langle Z\left|a_{i}^{+} a_{i}\right| Z\right\rangle=\left|z_{i}\right|^{2}$ and $\left\langle\xi\left|a_{i}^{+} a_{i}\right| \xi\right\rangle=\mathcal{N}\left|\xi_{i}\right|^{2}$, respectively, momentum-like states $|V\rangle$ and $|\alpha\rangle$ provide information on the $k$-mode boson population by means of $\left\langle V\left|b_{k}^{+} b_{k}\right| V\right\rangle=\left|v_{k}\right|^{2}$ and $\left\langle\alpha\left|b_{k}^{+} b_{k}\right| \alpha\right\rangle=\mathcal{N}\left|\alpha_{k}\right|^{2}$, respectively. The total boson number $\mathcal{N}$ is unchanged being $\langle Z|N| Z\rangle=\langle V|N| V\rangle$ and $\langle\xi|N| \xi\rangle=\langle\alpha|N| \alpha\rangle$.

As an application of the duality property of $|\xi\rangle$, we show that states $\left|S_{k}\right\rangle$, describing Schrödinger cats, can be defined having specific momentum properties. To this end an important preliminary condition consists in showing that $\mathcal{N}$-boson states $|\xi(\ell)\rangle$ with 
$\ell \in[1, M]$ can be exploited quite easily to form sets of $M$ orthogonal states. Recalling that the scalar product of two $\mathrm{SU}(\mathrm{M}) \mathrm{CS}$ is given by $\langle\eta \mid \xi\rangle=\left(\sum_{j} \eta_{j}^{*} \xi_{j}\right)^{\mathcal{N}}$, one has

$$
\langle\xi(h) \mid \xi(\ell)\rangle=\left(\sum_{j} \xi_{j}^{*}(h) \xi_{j}(\ell)\right)^{\mathcal{N}}=\delta_{h \ell} \quad \Leftrightarrow \quad \sum_{j} \xi_{j}^{*}(h) \xi_{j}(\ell)=\delta_{h \ell}
$$

which displays how the desired orthogonality directly ensues from the orthogonality of complex vectors $\vec{\xi}(\ell)=\left(\xi_{2}(\ell), \xi_{2}(\ell), \ldots\right)$ with $\ell \in[1, M]$. For fully localized states $|\xi(\ell)\rangle$ characterized by $\xi_{j}(\ell) \equiv \delta_{j \ell}$, the orthogonality condition $\langle\xi(h) \mid \xi(\ell)\rangle=\delta_{h \ell}$ is manifest. In the general case, however, the condition $\sum_{j} \xi_{j}^{*}(h) \xi_{j}(\ell)=\delta_{h \ell}$ with $\left|\xi_{\ell}(\ell)\right|>>\left|\xi_{j}(\ell)\right|$ can be achieved by exploiting the arbitrariness of the phases of $\xi_{j}(\ell)$. States $|\xi(\ell)\rangle$ describing strong boson localization have been employed to realize Schrödiger-cat states $\left|S_{k}\right\rangle$ in a ring of attractive bosons [25]. These were proven to well approximate the lowenergy states including the ground state in the regime of strong interaction. Following the recipe given in [25] we define $\left|S_{k}\right\rangle$ as a superposition of equal-weight localized states

$$
\left|S_{k}\right\rangle=\sum_{\ell=1}^{M} \frac{e^{i \tilde{k} \ell}}{\sqrt{M}}|\xi(\ell)\rangle, \quad\left|\xi_{\ell}(\ell)\right|>>\left|\xi_{j}(\ell)\right|, \quad j \neq \ell .
$$

As a consequence of the orthogonality of states $|\xi(\ell)\rangle$ states $\left|S_{k}\right\rangle$ appear themselves to be orthogonal namely $\left\langle S_{q} \mid S_{k}\right\rangle=\delta_{q k}$. We observe that, if $\left\langle\xi(\ell)\left|n_{i}\right| \xi(\ell)\right\rangle=\mathcal{N}\left|\xi_{i}(\ell)\right|^{2} \simeq \mathcal{N} \delta_{i \ell}$ evidences the information about boson localization at the $\ell$ th site, the expectation value

$$
\left\langle S_{k}\left|n_{i}\right| S_{k}\right\rangle=\sum_{h=1}^{M} \sum_{\ell=1}^{M} \frac{e^{i(\tilde{k} \ell-\tilde{q} h)}}{M}\left\langle\xi(h)\left|n_{i}\right| \xi(\ell)\right\rangle=\frac{\mathcal{N}}{M}, \quad \forall i,
$$

obtained through the properties $\left\langle\eta\left|a_{m}^{+} a_{i}\right| \xi\right\rangle=\mathcal{N} \eta_{m}^{*} \xi_{i}\langle\eta \mid \xi\rangle^{1-1 / \mathcal{N}}$ and $\langle\xi(h) \mid \xi(\ell)\rangle=\delta_{h \ell}$, confirms the the expected feature of full delocalization typical of Schroedinger states. We note how the possibility of constructing set of orthogonal trial states is quite important for applications to boson lattice systems such as model 3. While trial states can be used for approximating in a reliable way sets of energy eigenstates, the possibility to make them mutually orthogonal certainly enriches the approximation with an important feature.

In order to show that states $\left|S_{k}\right\rangle$ have specific momentum properties we rewrite $|\xi(\ell)\rangle$ in its dual form $|\alpha(\ell)\rangle$. Thanks to formula $25|\xi(\ell)\rangle=|\alpha(\ell)\rangle$ with $\alpha_{k}(\ell)=$ $\sum_{j=1}^{M} e^{-i \tilde{k} j} \xi_{j}(\ell) / \sqrt{M}$, one obtains $\langle\vec{p} \mid \xi(\ell)\rangle=\langle\vec{p} \mid \alpha(\ell)\rangle=C_{\vec{p}}(\mathcal{N}) \alpha_{1}^{p_{1}} \ldots \alpha_{M}^{p_{M}}$ giving

$$
\left\langle\vec{p} \mid S_{q}\right\rangle=\frac{1}{\sqrt{M}} \sum_{\ell} e^{i \ell \tilde{q}}\langle\vec{p} \mid \xi(\ell)\rangle=\frac{1}{\sqrt{M}} \sum_{\ell} e^{i \ell \tilde{q}} C_{\vec{p}}(\mathcal{N}) \alpha_{1}^{p_{1}}(\ell) \ldots \alpha_{M}^{p_{M}}(\ell) .
$$

In case of strong localization condition $\left|\xi_{\ell}(\ell)\right| \simeq 1>>\left|\xi_{j}(\ell)\right|$ leads to the approximation $\alpha_{k}(\ell) \simeq e^{-i \tilde{k} \ell} / \sqrt{M}$ and, in particular, to

$$
\left\langle\vec{p} \mid S_{q}\right\rangle \simeq \frac{1}{\sqrt{M}} \sum_{\ell} e^{i \ell \tilde{q}} C_{\vec{p}}(\mathcal{N}) \frac{e^{-i \ell \sum_{k} p_{k} \tilde{k}}}{M^{\sum_{k} p_{k} / 2}}=\frac{\sqrt{\mathcal{N} !}}{M^{(\mathcal{N}+1) / 2}} e^{-\frac{1}{2} \sum_{k} \ln \left(p_{k} !\right)} \sum_{\ell} e^{i \frac{2 \pi \ell}{M}[q-\lambda(\vec{p})]}
$$


where $C_{\vec{p}}(\mathcal{N}):=\left[\mathcal{N} ! / \prod_{k} p_{k} !\right]^{1 / 2}$ and $\lambda(\vec{p})=\sum_{k} k p_{k}=0,1,2, \ldots \bmod (M)$. The latter represents the eigenvalue of the total quasi-momentum operator $P=\sigma \sum_{k} k b_{k}^{+} b_{k}$ with $\sigma=2 \pi / M$ such that $P|\vec{p}\rangle=\sigma \lambda(\vec{p})|\vec{p}\rangle$. It is worth recalling that, in the discrete geometry of ring lattices, the quasi-momentum properties are described through the displacement operator $D=\exp [-i \sigma P]$, whose action is displayed by $D a_{\ell} D^{+}=a_{\ell+1}$ and $D b_{k} D^{+}=b_{k} e^{i k \sigma}$. Based on equation $D|\vec{p}\rangle=e^{-i \sigma \lambda(\vec{p})}|\vec{p}\rangle$ Fock states can be organized in $M$ equivalence classes labeled by $\lambda(\vec{p})$. Index $q$ in $\left|S_{q}\right\rangle$ therefore characterizes the quasimomentum associated with $\left|S_{q}\right\rangle$ since the term $\sum_{\ell} \exp [i \ell[\tilde{q}-\lambda(\vec{p})]]$ in $\left\langle\vec{p} \mid S_{q}\right\rangle$ vanishes whenever $|\vec{p}\rangle$ has a momentum $\lambda(\vec{p}) \neq q \bmod (M)$. States $\left|S_{q}\right\rangle$, in the presence of strong localization, supply a set of $M$ orthogonal states whose label $q$ bears information on the class with quasi-momentum $\lambda$ maximally involved in the realization of $\left|S_{q}\right\rangle$, within the $\mathcal{N}$-particle Hilbert space. As for Glauber-like states, we note that $\langle X \mid Z\rangle=$ $\prod_{j}\left\langle x_{j} \mid z_{j}\right\rangle=\prod_{j} \exp \left[\bar{x}_{j} z_{j}-\left(\left|z_{j}\right|^{2}+\left|x_{j}\right|^{2}\right) / 2\right]$ so that $|Z\rangle$ and $|X\rangle$ cannot be orthogonal. At most, $M$ quasi-orthogonal states can be obtained by considering sets $\left\{x_{j}(\ell)\right\}$ such that $\left|x_{j}(\ell)\right|^{2} \simeq \mathcal{N} \delta_{j \ell}$ for which $|\langle X(h) \mid X(\ell)\rangle| \simeq e^{-\mathcal{N}}$. Representation of Schrödinger-cat states in terms of quasi-orthogonal states $|Z\rangle$ can be developed under these conditions.

\section{Conclusions}

In this paper we have compared variational schemes based on trial states $|F\rangle,|Z\rangle$ and $|\xi\rangle$, widely used in applications to many-mode boson systems. To illustrate their distinctive features we have applied such schemes to the BH model which has became, in the recent years, the paradigm of real interacting-boson lattice systems. Such a comparison has been aimed at evidencing the specific characters of each scheme to favour their applications in the study of the properties of many-mode boson systems.

In section 2, we have applied the $|F\rangle$-based scheme to the BH model showing, within the corresponding dynamical scenario, that collective variables $\alpha_{i}, \alpha_{i}^{*}$ form a (classical) Weyl-Heisenberg sub-algebra in the Poisson algebra of variables $f_{n}^{j}, \bar{f}_{m}^{\ell}$. This crucial property allows reduction of the $|F\rangle$-based picture, exhibiting a more pronounced quantum character, to the $|Z\rangle$-based picture based on Glauber's CS. The semiclassical character of the latter appears to be an effective, dynamically-consistent procedure incorporating the Bogoliubov approximation.

In section 3, we have shown that Glauber-like trial state $|Z\rangle$ is a superposition of $\mathrm{SU}(\mathrm{M}) \mathrm{CS}|\mathcal{N}, \xi\rangle$ that involves all the $\mathcal{N}$-particle sectors of the Hilbert space. We have exploited this property to explain why the dynamical equations relevant to the $\mathrm{BH}$ model obtained in the $|\mathcal{N}, \xi\rangle$-based scheme coincide with the equations derived in $|Z\rangle$-based scheme. The meaning of microscopic CS parameters of such schemes has been illustrated and related to the fact that states $|\mathcal{N}, \xi\rangle$ are boson-number preserving. Also, in section 3, we have discussed explicitly the procedure that enables one to recast state 2 into the standard form $|\xi\rangle=g|\Omega\rangle$ of CS theory involving the extremal vector $|\Omega\rangle$.

Section 4 has been devoted to discuss the duality property of space-like states

$|Z\rangle$ and $|\xi\rangle$ which allows to rewrite them as momentum-like states involving modes 
$b_{k}^{+}$. This property has been used for constructing Schrödinger-cat states with specific momentum features for bosons in ring lattices. In general, the use of states $|\xi\rangle$ and the possibility to construct set of orthogonal states outlined in section 4 should allow a better characterization of low-energy regimes in systems of bosons confined in ring lattices whose standard description is given in terms of Hamiltonian 3, Particularly, the duality property of states $|Z\rangle$ and $|\xi\rangle$ finds a natural application in the study of supercurrents and vortex states occurring in such systems [22], [23].

As observed in section 1, while the $|Z\rangle$-based scheme has been extensively used in applications, the interest for the $|\xi\rangle$ and $|F\rangle$-based schemes is more recent. The more pronounced quantum character of the $|F\rangle$-based scheme is expected to supply, in the applications to BH-like models, a better description of the critical behaviors [29]-[32] inherent in quantum phase transitions. For the same reason it should supply an effective tool to study the complex dynamics [27], [28] of bosons in lattice systems. Bosons distributed in small arrays (and thus involving small number $M$ of space modes) are specially interesting since they can switch from fully quantum to intermediate semiclassical behaviors by adjusting model parameters [23]-[25]. The correspondingly small number of components $\left|F_{i}\right\rangle$ in $|F\rangle$ makes it feasible for performing numerical simulations of equations 8. These aspects will be investigated in a separate paper [43].

\section{Appendix A. Application of formula 2 to the case $\mathrm{M}=2$}

It is quite easy to show that formula 2 with $M=2$ reproduces the standard grouptheoretic definition of $\mathrm{SU}(2)$ coherent state. In this case $A^{+}=\xi_{1} a_{1}^{+}+\xi_{2} a_{2}^{+}$. Then

$$
\begin{gathered}
|\mathcal{N}, \xi\rangle=(\mathcal{N} !)^{-1 / 2}\left(A^{+}\right)^{\mathcal{N}}|0,0\rangle=\frac{1}{\sqrt{\mathcal{N} !}} \sum_{s=0}^{\mathcal{N}} \frac{\mathcal{N} ! \xi_{2}^{s} \xi_{1}^{\mathcal{N}-s}}{s !(\mathcal{N}-s) !}\left(a_{2}^{+}\right)^{s}\left(a_{1}^{+}\right)^{\mathcal{N}-s}|0,0\rangle \\
=\sum_{s=0}^{\mathcal{N}} C_{s}(\mathcal{N}) \xi_{2}^{s} \xi_{1}^{\mathcal{N}-s}|\mathcal{N}-s, s\rangle=e^{i \mathcal{N} \phi_{1}} \sum_{s=0}^{\mathcal{N}} \frac{C_{s}(\mathcal{N}) z^{s}}{\left(1+|z|^{2}\right)^{\mathcal{N} / 2}}|J ;-J+s\rangle=e^{i \mathcal{N} \phi_{1}}|J ; z\rangle
\end{gathered}
$$

where $C_{s}(\mathcal{N}) \equiv \sqrt{\mathcal{N} !} / \sqrt{s !(\mathcal{N}-s) !}$, the definition $z=\xi_{2} / \xi_{1}$ has been used, and $\phi_{\ell}$ is the phase of $\xi_{\ell}$. Moreover, $|J ;-J+s\rangle \equiv|\mathcal{N}-s, s\rangle$, where $J=\mathcal{N} / 2$, can be seen as the $m$ th vector (with $m=-J+s$ ) in the standard basis $\left\{|J ; m\rangle: J_{3}|J ; m\rangle=m|J ; m\rangle\right\}$ of algebra $\operatorname{su}(2)$ within the Schwinger boson picture of spin operators $J_{3}=\left(a_{2}^{+} a_{2}-a_{1}^{+} a_{1}\right) / 2$, $J_{+}=a_{2}^{+} a_{1}=\left(J_{-}\right)^{+}$. State $g|\Omega\rangle=e^{\zeta J_{+}-\zeta^{*} J_{-}}|J ;-J\rangle=\left(1+|z|^{2}\right)^{-J} e^{z J_{+}}|J ;-J\rangle$, obtained through the standard decomposition [2] $g=e^{\zeta J_{+}-\zeta^{*} J_{-}}=e^{z J_{+}} e^{J_{3} \ln \left(1+|z|^{2}\right)} e^{-z^{*} J_{-}}$, coincides with state $|J ; z\rangle$ just defined, where $z$ and $\zeta$ have the same phase and $|\zeta|=\operatorname{tg}|z|$.

\section{Appendix B. Derivation of dynamical equations for $z_{j}$}

Based on dynamical equations 8 governing the evolution of variables $f_{m}^{i}$ one has

$$
i \frac{d \alpha_{i}}{d t}=\sum_{m} \sqrt{m+1}\left[i \frac{d \bar{f}_{m}^{i}}{d t} f_{m+1}^{i}+i \frac{d f_{m+1}^{i}}{d t} \bar{f}_{m}^{i}\right]
$$


Some remarks on the coherent-state variational

$$
\begin{gathered}
=\sum_{m} \sqrt{m+1}\left[f_{m+1}^{i}\left(-\frac{U}{2}\left(m^{2}-m\right) \bar{f}_{m}^{i}+\sqrt{m+1} \bar{f}_{m+1}^{i} \Phi_{i}+\sqrt{m} \bar{f}_{m-1}^{i} \Phi_{i}^{*}\right)+\right. \\
\left.+\bar{f}_{m}^{i}\left(\frac{U}{2}\left(m^{2}+m\right) f_{m+1}^{i}-\sqrt{m+2} f_{m+2}^{i} \Phi_{i}^{*}-\sqrt{m+1} f_{m}^{i} \Phi_{i}\right)\right] \\
=\sum_{m} U m \sqrt{m+1} \bar{f}_{m}^{i} f_{m+1}^{i}+\Phi_{i}\left[\sum_{m}\left((m+1) \bar{f}_{m+1}^{i} f_{m+1}^{i}-m \bar{f}_{m}^{i} f_{m}^{i}\right)-\sum_{m} \bar{f}_{m}^{i} f_{m}^{i}\right]+ \\
+\Phi_{i}^{*}\left(\sum_{m} \sqrt{m+1} \sqrt{m} \bar{f}_{m-1}^{i} f_{m+1}^{i}-\sum_{m} \sqrt{m+1} \sqrt{m+2} \bar{f}_{m}^{i} f_{m+2}^{i}\right)
\end{gathered}
$$

where the index- $m$ range is $[0, \infty]$. Thus $\dot{\alpha}_{i}$ is formed by three terms. Substitution $m \rightarrow$ $m+1$ in the first summation of the third term (one should notice that $\bar{f}_{-1}^{i}=0$ ) shows that the latter vanishes, while, in the second term, $\Sigma_{m}\left[(m+1) \bar{f}_{m+1}^{i} f_{m+1}^{i}-m \bar{f}_{m}^{i} f_{m}^{i}\right]=0$ is easily proven. Further simplification is achieved if the the on-site normalization condition $\left\langle F_{i} \mid F_{i}\right\rangle=\sum_{m} \bar{f}_{m}^{i} f_{m}^{i}=1$ is imposed. Notice that choice 9 authomatically ensures such a condition since $\left|F_{i}\right\rangle=\left|z_{i}\right\rangle$ and Glauber CS are such that $\left\langle z_{i} \mid z_{i}\right\rangle=1$. Under this circumstance the second term reduces to $\Phi_{i}$.

\section{Appendix C. Conservation of $\mathcal{N}$ and other constants of motion}

After recalling that $\mathcal{N}=\langle\Psi|N| \Psi\rangle=\left\langle\Psi\left|\sum_{j} n_{j}\right| \Psi\right\rangle=\sum_{j} \sum_{n} n\left|f_{n}^{j}\right|^{2}$ where $\left\langle\Psi\left|n_{j}\right| \Psi\right\rangle=$ $\sum_{n} n\left|f_{n}^{j}\right|^{2}$ and $n \in[0, \infty], j \in[1, M]$, let us consider the Poisson bracket of $\mathcal{N}$ and $\mathcal{H}$

$$
\begin{gathered}
\{\mathcal{N}, \mathcal{H}\}=\sum_{j} \sum_{n} n\left\{\left|f_{n}^{j}\right|^{2}, \mathcal{H}\right\}=\sum_{j} \sum_{n} n\left[\bar{f}_{n}^{j}\left\{f_{n}^{j}, \mathcal{H}\right\}+f_{n}^{i}\left\{\bar{f}_{n}^{i}, \mathcal{H}\right\}\right]= \\
=-i \sum_{j} \sum_{n} n\left[\bar{f}_{n}^{j}\left(\frac{U}{2}\left(n^{2}-n\right) f_{n}^{j}-\sqrt{n+1} f_{n+1}^{j} \Phi_{j}^{*}-\sqrt{n} f_{n-1}^{j} \Phi_{j}\right)\right. \\
\left.\quad+f_{n}^{j}\left(-\frac{U}{2}\left(n^{2}-n\right) \bar{f}_{n}^{j}+\sqrt{n+1} \bar{f}_{n+1}^{j} \Phi_{j}+\sqrt{n} \bar{f}_{n-1}^{j} \Phi_{j}^{*}\right)\right] \\
=-i \sum_{j} \Phi_{j}^{*} \sum_{n}\left(\sqrt{n+1} f_{n+1}^{j} \bar{f}_{n}^{j}\right)-i \sum_{j} \Phi_{j} \sum_{n}\left(-\sqrt{n+1} \bar{f}_{n+1}^{j} f_{n}^{j}\right) \\
=-i \sum_{j} \Phi_{j}^{*} \alpha_{j}-\sum_{j} \Phi_{j} \alpha_{j}^{*}=-i \sum_{j} \alpha_{j} \sum_{\ell \in j}\left(T_{\ell j} \alpha_{\ell}^{*}-T_{\ell j} \alpha_{\ell}\right)=0 .
\end{gathered}
$$

Then $d \mathcal{N} / d t=\{\mathcal{N}, \mathcal{H}\}=0$. A similar calculation allows one to evidence that other $M$ constants of motion are involved in the dynamics of $f_{n}^{j}$. These are $I_{j}=\sum_{n}\left|f_{n}^{j}\right|^{2}$ for which $\left\{\mathcal{I}_{j}, \mathcal{H}\right\}=0$. Quantities $I_{j}$ are in involution with $\mathcal{N}, \alpha_{i}$ and $I_{i}$ namely $\left\{I_{j}, \mathcal{N}\right\}=0$ and $\left\{I_{j}, \alpha_{i}\right\}=0 \forall j$. One easily check as well that $\left\{I_{j}, I_{i}\right\}=0$ for each $i$ and $j$.

\section{Appendix D. Formulas relevant to the SU(M)-CS picture}

In order to obtain $\mathcal{L}(\xi)=i \hbar\left\langle\xi\left|\partial_{t}\right| \xi\right\rangle-\langle\xi|H| \xi\rangle$ one needs to calculate $\left\langle\xi\left|\partial_{t}\right| \xi\right\rangle$ and $\left\langle\xi\left|\left(n_{i}-1\right) n_{i}\right| \xi\right\rangle$ in $\mathcal{H}(\xi)=\langle\xi|H| \xi\rangle$. Concerning $\left\langle\xi\left|\left(n_{i}-1\right) n_{i}\right| \xi\right\rangle$, (recall that $|\xi\rangle \equiv|S ; \xi\rangle$ ) one should observe that $\left[a_{i},\left(A^{+}\right)^{s}\right]=s \xi_{i}\left(A^{+}\right)^{s-1}$, and that

$$
a_{i}|\xi\rangle=a_{i} \rho_{S}\left(A^{+}\right)^{S}|0\rangle=\rho_{S}\left[\left(A^{+}\right)^{S} a_{i}+S \xi_{i}\left(A^{+}\right)^{S-1}\right]|0\rangle=\sqrt{S} \xi_{i}\left|\xi^{\prime}\right\rangle
$$


where $\rho_{S}=1 / \sqrt{S !}$, and $\left|\xi^{\prime}\right\rangle=\rho_{S-1}\left(A^{+}\right)^{S-1}|0\rangle$ is a $(S-1)$-boson coherent state. Iterating this calculation gives $a_{i}^{2}|\xi\rangle=\xi_{i}^{2} \sqrt{S(S-1)}\left|\xi^{\prime \prime}\right\rangle$ with $\left|\xi^{\prime \prime}\right\rangle=\rho_{S-2}\left(A^{+}\right)^{S-2}|0\rangle$. Therefore $\left\langle\xi\left|n_{i}\right| \xi\right\rangle=\ldots=N\left|\xi_{i}\right|^{2}$ whereas

$$
\left\langle\xi\left|\left(n_{i}-1\right) n_{i}\right| \xi\right\rangle=\left\langle\xi\left|\left(a_{i}^{+}\right)^{2} a_{i}^{2}\right| \xi\right\rangle=S(S-1)\left|\xi_{i}\right|^{4}\left\langle\xi^{\prime \prime} \mid \xi^{\prime \prime}\right\rangle=S(S-1)\left|\xi_{i}\right|^{4} .
$$

Similarly, one finds $\left\langle\xi\left|a_{m}^{+} a_{i}\right| \xi\right\rangle=\left\langle 0\left|\rho_{S} A^{S} a_{m}^{+} a_{i} \rho_{S}\left(A^{+}\right)^{S}\right| 0\right\rangle=S \xi_{m}^{*} \xi_{i}\left\langle\xi^{\prime} \mid \xi^{\prime}\right\rangle=S \xi_{m}^{*} \xi_{i}$. For two generic states $|\eta\rangle$, $|\xi\rangle$ the latter becomes $\left\langle\eta\left|a_{m}^{+} a_{i}\right| \xi\right\rangle=S \eta_{m}^{*} \xi_{i}\left(\sum_{i} \eta_{i}^{*} \xi_{i}\right)^{S-1}$ where the inner product $\langle\eta \mid \xi\rangle=\left(\sum_{i} \eta_{i}^{*} \xi_{i}\right)^{S}$ of two $S$-boson states $|\eta\rangle$, $|\xi\rangle$ has been used. In the effective Lagrangian $\mathcal{L}$ term $\left\langle\xi\left|\partial_{t}\right| \xi\right\rangle$ can be recast as

$$
\begin{gathered}
\left\langle\xi\left|\partial_{t}\right| \xi\right\rangle=\left\langle\xi\left|\sum_{j} \dot{\xi}_{j} \partial_{\xi_{j}}\right| \xi\right\rangle=\rho_{S-1}^{2}\left\langle 0\left|A^{S-1} \sum_{j} \dot{\xi}_{j} A a_{j}^{+}\left(A^{+}\right)^{S-1}\right| 0\right\rangle=\left\langle\xi^{\prime}\left|\sum_{j} \dot{\xi}_{j} A a_{j}^{+}\right| \xi^{\prime}\right\rangle \\
=\left\langle\xi^{\prime}\left|\sum_{j} \dot{\xi}_{j}\left[a_{j}^{+} A+\xi_{j}^{*}\right]\right| \xi^{\prime}\right\rangle=\sum_{j} \dot{\xi}_{j} \xi_{j}^{*}+\sum_{j} \sum_{m} \xi_{m}^{*} \dot{\xi}_{j}\left\langle\xi^{\prime}\left|a_{j}^{+} a_{m}\right| \xi^{\prime}\right\rangle \\
=\sum_{j} \dot{\xi}_{j} \xi_{j}^{*}+\sum_{j} \sum_{m} \xi_{m}^{*} \dot{\xi}_{j}(S-1) \xi_{m} \xi_{j}^{*}=\sum_{j} \dot{\xi}_{j} \xi_{j}^{*}+(S-1) \sum_{j} \dot{\xi}_{j} \xi_{j}^{*}=S \sum_{j} \dot{\xi}_{j} \xi_{j}^{*} .
\end{gathered}
$$

Concluding, the four/two-boson expectation values just obtained provide the effective Hamiltonian $\langle\xi|H| \xi\rangle=\frac{U}{2} S(S-1) \sum_{j}\left|\xi_{j}\right|^{4}-S \sum_{<j, \ell>} T_{j \ell} \xi_{j}^{*} \xi_{\ell}$ occuring in $\mathcal{L}(\xi)$.

\section{Appendix E. Derivation of operator $A^{+}$}

After setting $\sum_{k=2}^{M} \theta_{k}^{2}:=\theta^{2}$, the action of $e^{i D}$ on $a_{1}^{+}$is given by the standard formula

$$
e^{i D} a_{1}^{+} e^{-i D}=\sum_{s=0}^{\infty} \frac{i^{s}}{s !}\left[D, a_{1}^{+}\right]_{s}
$$

where $\left[D, a_{1}^{+}\right]_{s}=\left[D,\left[D, a_{1}^{+}\right]_{s-1}\right],\left[D, a_{1}^{+}\right]_{1}=\left[D, a_{1}^{+}\right]$, and $\left[D, a_{1}^{+}\right]_{0}=\mathbf{1}$. Observing that $\left[D, a_{1}^{+}\right]_{2 r}=\theta^{2 r} a_{1}^{+}$and $\left[D, a_{1}^{+}\right]_{2 r+1}=\theta^{2 r} Q$ with $Q=\sum_{k=2}^{M} \theta_{k} a_{k}^{+}$, one obtains

$$
e^{i D} a_{1}^{+} e^{-i D}=\sum_{r=0}^{\infty} \frac{(-)^{r}}{(2 r) !} \theta^{2 s} a_{1}^{+}+\sum_{r=0}^{\infty} \frac{i(-)^{r}}{(2 r+1) !} \theta^{2 s} Q=a_{1}^{+} \cos \theta+i Q \frac{\sin \theta}{\theta}=\sum_{j=1}^{M} y_{j} a_{j}^{+}
$$

where $y_{1}=\cos \theta$ and $y_{k}=i \theta_{k} \sin \theta / \theta$ if $k \neq 1$.

\section{Appendix F. Two-boson operators of algebra su(M)}

The fact that $T(\zeta) \in \mathrm{SU}(\mathrm{M})$ is easily demonstrated by recalling that, within a Schwingerlike picture, algebra $\mathrm{su}(\mathrm{M})$ can be realized in terms of two-boson operators $a_{j}^{+} a_{k}, a_{k}^{+} a_{j}$ with $1 \leq j \leq M-1$ and $j+1 \leq k \leq M$ that play the role of lowering and raising operators, respectively. This set is completed by the generators of the Cartan-subalgebra $\left\{h_{k}, k=2, \ldots M:\left[h_{k}, h_{\ell}\right]=0\right\}$ where each of the $M-1$ operators $h_{k}$ can be written as an appropriate linear combinations of number operators $n_{i}=a_{i}^{+} a_{i}, i=1,2 \ldots, M$. We notice that, consistent with the presence of the group-invariant operator $N=\sum_{i=1}^{M} n_{i}$, 
only $M-1$ operators $h_{k}$ can be realized with $M$ operators $n_{i}$. A generic element of $\mathcal{G}$ $=\operatorname{su}(\mathrm{M})=\left\{a_{j}^{+} a_{m}(m \neq j): m, j \in[1, M] ; h_{k}: k \in[2, M]\right\}$ is thus given by

$$
\sum_{j=1}^{M-1} \sum_{k=j+1}^{M}\left(z_{k j} a_{j}^{+} a_{k}+z_{k j}^{*} a_{j} a_{k}^{+}\right)+\sum_{k=2}^{M} \alpha_{k} h_{k}
$$

where $z_{k j}=x_{k j}+i y_{k j}$ and $x_{k j}, y_{k j}, \alpha_{k} \in \mathbf{R}$. Since elements $g \in G$ of a Lie group $G$ are generated by the Lie algebra element $a \in \mathcal{G}=\operatorname{Lie}(G)$ through the exponential map $g=\exp (i a)$ then the latter fromula shows that $T(\zeta) \in \mathrm{SU}(\mathrm{M})$.

\section{References}

[1] Perelomov A 1986 Generalized Coherent States and Their Applications (Berlin: Springer)

[2] Zhang W M, Feng D H, and Gilmore R 1990 Rev. Mod. Phys. 62, 867

[3] Solomon A I, Feng Y, and Penna V 1999 Phys. Rev. B 603044

[4] Nemoto K, Holmes C A, Milburn G J, and Munro W J 2000 Phys. Rev. A 63013604

[5] Franzosi R, Penna V, Phys. Rev. A 65, 013601 (2002)

[6] Amico L and Penna V 2000 Phys. Rev. B 621224

[7] Burnett K, Edwards M, Clark C W, and Shotter M 2002 J. Phys. B: At. Mol. Opt. Phys. 351671

[8] Polkovnikov A, Sachdev S, and Girvin S M 2002 Phys. Rev. A 66053607

[9] Franzosi R, Penna V, and Zecchina R 2000 Int. J. Mod. Phys. B 14943

[10] Kuriyama A, Yamamura M, Providencia C, da Providencia J, and Tsue Y 2003 J. Phys. A: Math. Gen. 3610361

[11] Jain P, and Gardiner C W 2004 J. Phys. B: At. Mol. Opt. Phys. 373649

[12] This mean-field scheme has been applied to quantum-soliton theory in Brown D W, Lindenberg K, and West B J 1986 Phys. Rev. A 334104

[13] Gilmore R, Bowden C M, and Narducci L M 1975 Phys. Rev. A 121019

[14] Arecchi F T, Courtens E, Gilmore R, and Thomas H 1972 Phys. Rev. A 62211

[15] Franzosi R 2007 Phys. Rev. A 75053610

[16] Buonsante P, Franco R, and Penna V 2005 J. Phys. A: Math. Gen. 388393

[17] Kolovsky A R 2007 Phys. Rev. Lett. 99020401

[18] Oelkers N, and Links J 2007 Phys. Rev. B 75115119

[19] Mossmann S and Jung C 2006 Phys. Rev. A 74033601

[20] Pando C L and Doedel E J 2005 Phys. Rev. E 71056201

[21] Gati R, and Oberthaler M K 2007 J. Phys. B: At. Mol. Opt. Phys. 40 R61

[22] Dunningham J, and Hallwood D 2006 Phys. Rev. A 74023601

[23] Lee C, Alexander T J, Kivshar Y S 2006 Phys. Rev. Lett. 97180408

[24] Stickney J A, Anderson D Z, and Zozulya A A 2007 Phys. Rev. A 75013608

[25] Buonsante P, Penna V, and Vezzani A 2005 Phys. Rev. A 72043620

[26] Gutzwiller M C 1963 Phys. Rev. 10159

[27] Jaksch D, Venturi V, Cirac J I, Williams C J, and Zoller P 2002 Phys. Rev. Lett. 89040402

[28] Damski B, Santos L, Tiemann E, Lewenstein M, Kotochigova S, Julienne P, and Zoller P 2003 Phys. Rev. Lett. 90110401

[29] Sheshadri K, Krishnamurthy H R, Pandit R, Ramakrishnan T V, 1993 Euorphys. Lett. 22257.

[30] Polkovnikov A, Altman E, Demler E, Halperin B, and Lukin M D 2005 Phys. Rev. A 71063613

[31] Menotti C, Trefzger C, Lewenstein M 2007 Phys. Rev. Lett. 98235301

[32] Buonsante P, Massel F, Penna V, and Vezzani A 2007 Laser Phys. 17538

[33] Even if $|Z\rangle$ and $|F\rangle$ do not conserve $N$, this property is recovered within the mean-field picture where $\langle\Phi|N| \Phi\rangle$ with $\Phi=Z, F$ is a constant of motion of the effective Hamiltonian $\mathcal{H}=\langle\Phi|H| \Phi\rangle$.

[34] Klauder J R and Skagerstam B S 1985 Coherent States (Singapore: World Scientific) 
[35] Nemoto K 2000 J. Phys. A: Math. Gen. 333493

[36] Zhang W M, Feng D H 1991 Phys. Rev. C 431127

[37] Rowe D J 2004 Nucl. Phys. A $\mathbf{7 4 5} 47$

[38] Daoud M 2004 Phys. Lett. A 329318

[39] Vourdas A 2006 J. Phys. A: Math. Gen. 39 R65

[40] Fisher M P A, Weichman P B, Grinstein G, and Fisher S D 1989 Phys. Rev. B 40546

[41] Johansson M 2004 J. Phys. A: Math. Gen. 372201

[42] Amico L, Osterloh A, and Cataliotti F 2005 Phys. Rev. Lett. 95063201

[43] P. Buonsante, V. Penna, and A. Vezzani, in preparation 